\title{
Immunoglobulin Classification Based on FC* and GC* Features
}

\author{
Hao Wan ${ }^{1}$, Jina Zhang ${ }^{2}$, Yijie Ding ${ }^{3}$, Hetian Wang ${ }^{4 \star}$ and Geng Tian ${ }^{2 *}$ \\ ${ }^{1}$ Institute of Advanced Cross-field Science, College of Life Science, Qingdao University, Qingdao, China, ${ }^{2}$ Geneis (Beijing) Co., \\ Ltd., Beijing, China, ${ }^{3}$ Yangtze Delta Region Institute (Quzhou), University of Electronic Science and Technology of China, Quzhou, \\ China, ${ }^{4}$ Beidahuang Industry Group General Hospital, Harbin, China
}

Immunoglobulins have a pivotal role in disease regulation. Therefore, it is vital to accurately identify immunoglobulins to develop new drugs and research related diseases. Compared with utilizing high-dimension features to identify immunoglobulins, this research aimed to examine a method to classify immunoglobulins and non-immunoglobulins using two features, $\mathrm{FC}^{\star}$ and $\mathrm{GC}^{\star}$. Classification of 228 samples (109 immunoglobulin samples and 119 non-immunoglobulin samples) revealed that the overall accuracy was $80.7 \%$ in 10-fold cross-validation using the $\mathrm{J} 48$ classifier implemented in Weka software. The FC* feature identified in this study was found in the immunoglobulin subtype domain, which demonstrated that this extracted feature could represent functional and structural properties of immunoglobulins for forecasting.

Edited by:

Quan Zou,

University of Electronic Science and Technology of China, China

Reviewed by:

Ru Xiaoqing,

University of Tsukuba, Japan

Pengfei Zhu,

Tianjin University, China

*Correspondence:

Hetian Wang

hetw2021@163.com

Geng Tian

tiang@geneis.cn

Specialty section:

This article was submitted to

Computational Genomics,

a section of the journal

Frontiers in Genetics

Received: 01 December 2021

Accepted: 22 December 2021

Published: 24 January 2022

Citation:

Wan $\mathrm{H}$, Zhang J, Ding $Y$, Wang $\mathrm{H}$ and Tian $G$ (2022) Immunoglobulin Classification Based on FC* and $G C^{\star}$ Features.

Front. Genet. 12:827161. doi: $10.3389 /$ fgene.2021.827161
Keywords: immunoglobulin classification, machine learning, key feature extraction, MRMD, autoprop

\section{INTRODUCTION}

Immunoglobulins, or antibodies, are a group of proteins secreted by B lymphocytes that recognize invading antigens and bind to antigens with high affinity and specificity to neutralize toxic substances. In general, antibodies are composed of two identical polypeptide chains, each with a light chain and a heavy chain (Narciso et al., 2011). They can be divided functionally into variable (V) domains, which bind to antigens, and constant $(\mathrm{C})$ domains, which activate, complement, or bind to Fc receptors (Schroeder and Cavacini, 2010). To predict the structure of immunoglobulins, (Lepore et al., 2017) developed the PIGSPro Server, an updated version of the popular PIGS Server.

Immunoglobulins have a pivotal role in disease regulation. Therefore, human and nonhuman polyclonal immunoglobulins have been used in therapeutics for many years. Five monoclonal immunoglobulins ranked in the top 10 blockbuster biotherapeutics drugs (Norman et al., 2020). Patients with primary immune deficiencies greatly benefit from the intravenous or subcutaneous administration of human immunoglobulin preparations (Perez et al., 2017). The advanced development of medicine is urged by its finite supply, which requires more identification of valuable therapeutic immunoglobulins. However, biochemical experiments are time-consuming with enzymes to fragment immunoglobulin molecules (Schroeder and Cavacini, 2010) or X-ray crystallography to obtain accurate structures (Narciso et al., 2011).

Machine learning can identify desired proteins from a large number of sequences within a short time to guide the experimental discovery process (Guo et al., 2020; Liu et al., 2020; Song G. et al., 2021; Cheng et al., 2021; Deng et al., 2021; Dong et al., 2021; Guo et al., 2021; Tang et al., 2021; Yu et al., 2021; Zhao et al., 2021). Over the past decades, researchers have developed many machine learning-based techniques for protein sequence analysis (Zhai et al., 2020; Zeng et al., 2020; Chen et al., 2021; Li et al., 2021). The bioinformatics approach of identifying immunoglobulins is to convert protein sequences into numerical vectors to reveal the internal structures of proteins. The 


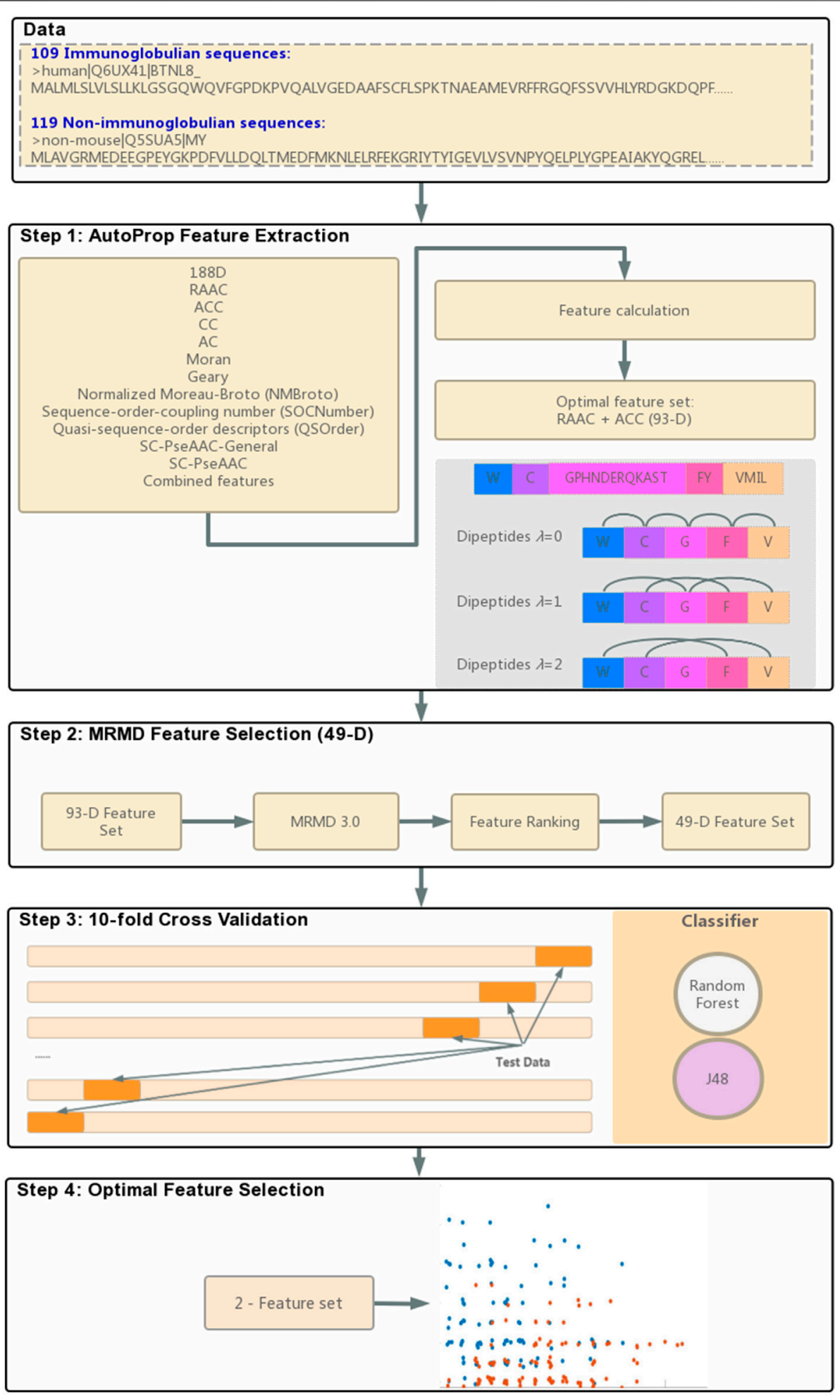

FIGURE 1 | Flowchart of identifying immunoglobulins. 
critical aspects of protein identification can be listed as follows: feature extraction, feature selection, and machine learning. Feature extraction methods include n-gram feature type: amino acid composition (AAC), Dipeptides (Dip), Tripeptides, where frequencies of $n$-length peptides are used as feature vectors (Ding et al., 2011; Gautam et al., 2013; Diener et al., 2016; Rahman et al., 2018; Liu et al., 2019; Lv et al., 2019; Fu et al., 2020; Wang H. et al., 2021; Wang J. et al., 2021; Zhai et al., 2020; Shao and Liu, 2021; Yang et al., 2021; Zhang et al., 2021). In addition, pseudo-amino acid composition (PseAAC) is also a widely adopted feature extraction method, including physicochemical properties between residues (Hansen et al., 2008; Sanders et al., 2011; Gautam et al., 2013; Chen et al., 2016; Diener et al., 2016; Khan et al., 2020; Awais et al., 2021; Naseer et al., 2021).

Many feature types and complex classification methods may generate redundant information (Song B. et al., 2021). Therefore, some studies began to eliminate redundant parts to improve the predictive performance of classification models. This process is also called feature selection. MRMD (Zou et al., 2016; Ao et al., 2020; Li et al., 2020a; Li et al., 2020b; Meng et al., 2020) and ANOVA (Anderson, 2001; Lv et al., 2019) are standard feature selection methods. For optimal feature identification, (Feng et al., 2021) uses the PCA and MCE methods to make the features orthogonal and obtain the core feature set with the minimum 10dimensional attributes for PPR gene identification and realized 97.9\% accuracy. (Li et al., 2020b) used a 19-dimensional feature model to classify anticancer peptide sequences. (Ao et al., 2020) used a 10-dimensional feature model to classify antioxidant proteins and realized $90.44 \%$ accuracy. (Meng et al., 2020) used a 6-dimensional feature model to classify cell wall lytic enzymes.

However, very few tools have been developed for immunoglobulin identification. (Tang et al., 2016) used the pseudo amino acid composition (PseAAC) feature extraction approach to realize over $96 \%$ prediction accuracy in their pioneering work on immunoglobulin identification. (Gong et al., 2021) used the CC-PSSM and monoTriKGap feature extraction, MRMD feature selection, and single dimension reduction methods to realize $92.1 \%$ immunoglobulin identification accuracy by two-dimensional features. However, the link between optimal features and functional structures of immunoglobulins remains to be investigated.

To obtain a diverse feature set, this study integrated 188-D physicochemical properties, auto-cross covariance (ACC) information, and dipeptide compositions of reduced amino acids. Dimensions were reduced using the max-relevancemax-distance (MRMD) method and the single dimension reduction method. The RF and J48 classifiers implemented in Weka software were used to identify immunoglobulins. Finally, two features can correctly predict immunoglobulins, $\mathrm{FC}^{\star}$ and $\mathrm{GC}^{\star}$. The entire modeling process is illustrated in Figure 1. The $\mathrm{FC}^{\star}$ feature identified in this study was found in immunoglobulin subtype domain IPR003599, which demonstrated that this extracted feature could represent functional and structural properties of immunoglobulins for forecasting.

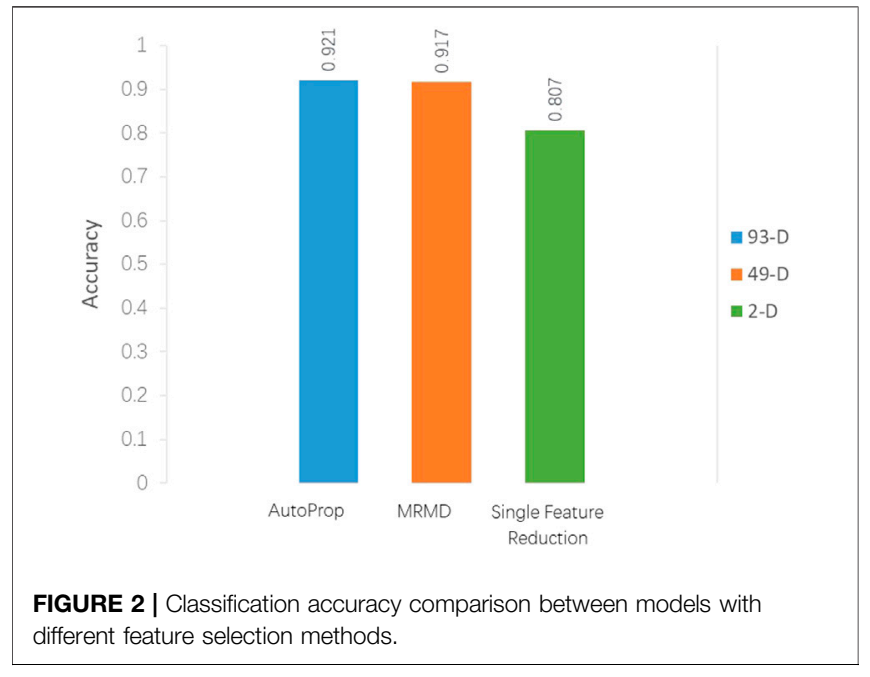

\section{MATERIALS AND METHODS}

\subsection{Datasets}

Data for this study were collected by (Tang et al., 2016), which contain 228 samples (109 immunoglobulin samples and 119 nonimmunoglobulin samples) extracted from the Universal Protein Resource (UniProt).

\subsection{RAAC}

Polypeptide chains fold to tertiary structures based on the physicochemical properties of residues (Tang et al., 2016). Analyzing the occurrence frequency of residue compositions cannot visualize three-dimensional protein structures. The reduced amino acid cluster (RAAC) method, replacing protein sequences with less than 20 amino acid alphabets based on a specific reducing scheme, can reduce sequence complexity. With removing non-essential information, functionally conserved regions will be displayed more clearly. Recent work presented 3D protein structures of ectonucleotide pyrophosphatase with a $1 \mathrm{D}$ view using the RAAC method (Solis, 2015; Zheng et al., 2019).

There are many choices of reduced schemes, and different decisions could produce distinctive protein classification results. For example, the RAACBook web server provided 74 types of reduced amino acid alphabets derived from over 1,000 published articles in PubMed (Zheng et al., 2019). Bins within the scheme are related to the chemical properties of amino acids. Dayhoff classes (AGPST, DENQ, HKR, ILMV, FWY, and C) are most used. Also, $\mathrm{S}$ and $\mathrm{T}$ are frequently together, and so are $\mathrm{K}$ and $\mathrm{R}, \mathrm{D}$, and E (Susko and Roger, 2007).

We used the AutoProp (Feng et al., 2020) to screen out the optimal reduced scheme of the immunoglobulin and nonimmunoglobulin sequences. GPHNDERQKAST, FY, VMIL, C, and W (Figure 1 Step 1) were used. Under this reduced scheme, the 20 amino acid alphabets were represented by five alphabets: $\mathrm{G}, \mathrm{F}, \mathrm{V}, \mathrm{C}$, and W. For instance, any amino acid that is a $\mathrm{G}, \mathrm{P}, \mathrm{H}$, $\mathrm{N}, \mathrm{D}, \mathrm{E}, \mathrm{R}, \mathrm{Q}, \mathrm{K}, \mathrm{A}, \mathrm{S}$, or T is then treated as character G. For any amino acid $\mathrm{F}$ and $\mathrm{Y}$, it is then treated as character $\mathrm{F}$, and so forth. 


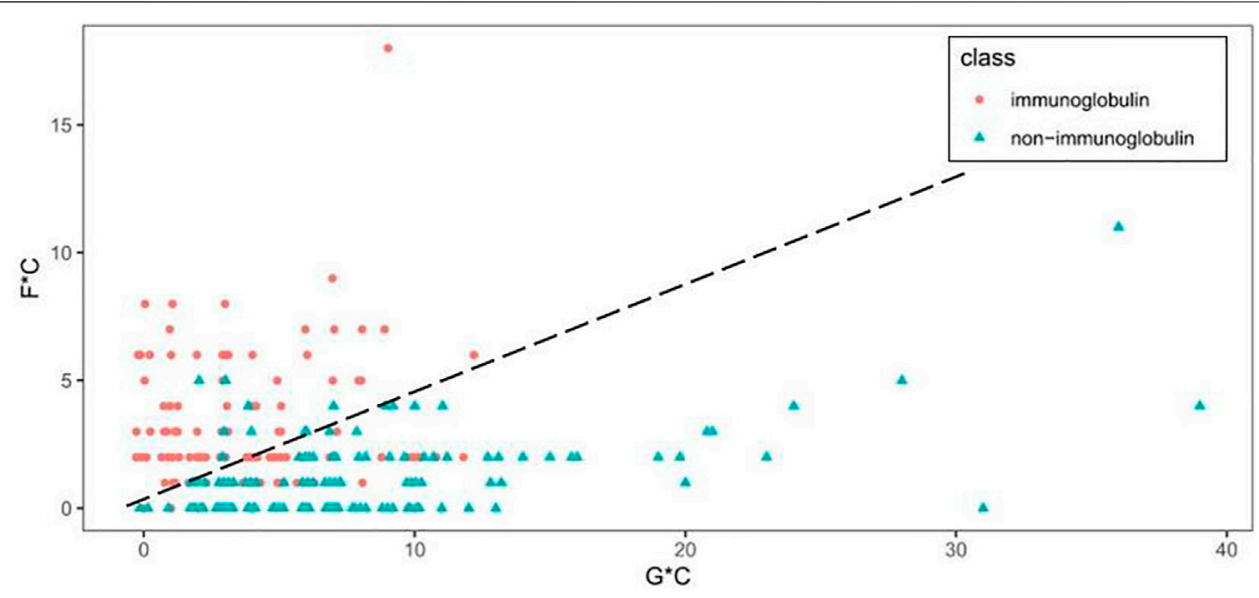

FIGURE 3 | Scatter plot of $\mathrm{GC}^{\star}$ and $\mathrm{FC}^{\star}$ features.

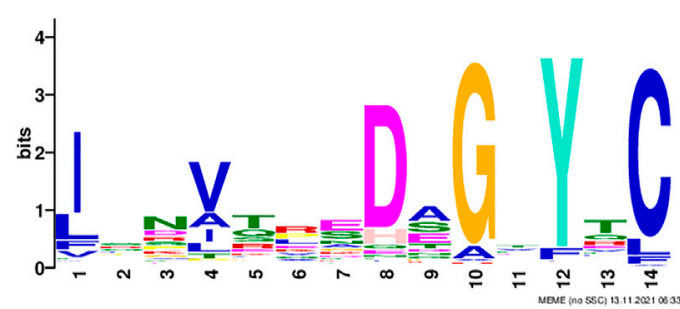

FIGURE 4 | Motif discovered among immunoglobulin sequences using the MEME tool; the height of the letter indicates its relative frequency at the given position within the motif.

\subsection{Feature Extraction}

A sequence can be represented by sequential form and discrete form. Homolog sequences can be compared with the BLAST or FASTA program benchmark datasets for traditional sequence comparison methods. However, the similarity-based way is unsuitable for distantly related sequences (Wei et al., 2014; Chen et al., 2016; Jin et al., 2019; Manavalan et al., 2019; Hong et al., 2020; Tang et al., 2020; Wang et al., 2020; Ding et al., 2021a; Ding et al., 2021b; Huang et al., 2021; Shao et al., 2021). By converting amino acid codes to a series of discrete numerical vectors, the discrete form can overcome this drawback and be used by machine learning for protein classification. Sometimes, proteins can be classified according to fewer features, while BLAST cannot.

Different numerical values of protein codes mean different feature descriptors. Feature descriptors provided by AutoProp include 188D, ACC, PseAAC, and another nine methods (Figure 1 Step 1). Also, AutoProp provides combined features between those methods. The built-in classifiers will then calculate the accuracy percentage of each feature and decide the optimal feature.

For our data, the optimal feature is the combined features of RAAC and ACC. RAAC features also represent dipeptides of reduced amino acid, like $\mathrm{CV}, \mathrm{C}^{\star} \mathrm{V}(\lambda$-gap $=1)$, and $\mathrm{C}^{\star *} \mathrm{~V}(\lambda$-gap $=$ 2 ). The following formula was used to calculate the values of those features:

$$
f_{u}=\frac{n_{u}^{\lambda}}{\sum n_{u}^{\lambda}},
$$

where $\lambda=0,1,2$, and $n_{u}^{\lambda}$ denotes the number of $\lambda$-gap dipeptides of type $u$ in a protein sequence.

ACC means the autocross covariance (ACC) transformation and contains auto covariance (AC) and cross-covariance (CC) and is introduced to transform protein sequences into fixedlength vectors (Feng et al., 2020). With its ability to identify sequence homologies, ACC has been successfully used for protein family classification and protein interaction prediction (Dong et al., 2009).

\subsection{MRMD}

The main disadvantage of the sequence word frequency vector is that they are usually huge. Therefore, dimension reduction, also called feature selection, is chosen for protein classification. The MRMD method, which is the maxrelevance-max-distance-based dimensionality reduction method, is more considered for relationships among features and stability of feature selection. Cross-validation and the ROC curve are usually used to evaluate classification accuracy. The MRMD method can reduce feature dimensions with few accuracy drops (Zou et al., 2016; He et al., 2020; Tao et al., 2020).

\subsection{Performance Measurement}

We used three metrics to evaluate model performance. Indicators include sensitivity (SE), specificity (SP), and Accuracy (Jiang et al., 2013; Wang X. et al., 2021). Calculation methods are described as follows:

$$
\mathrm{SE}=\frac{T P}{T P+F N},
$$




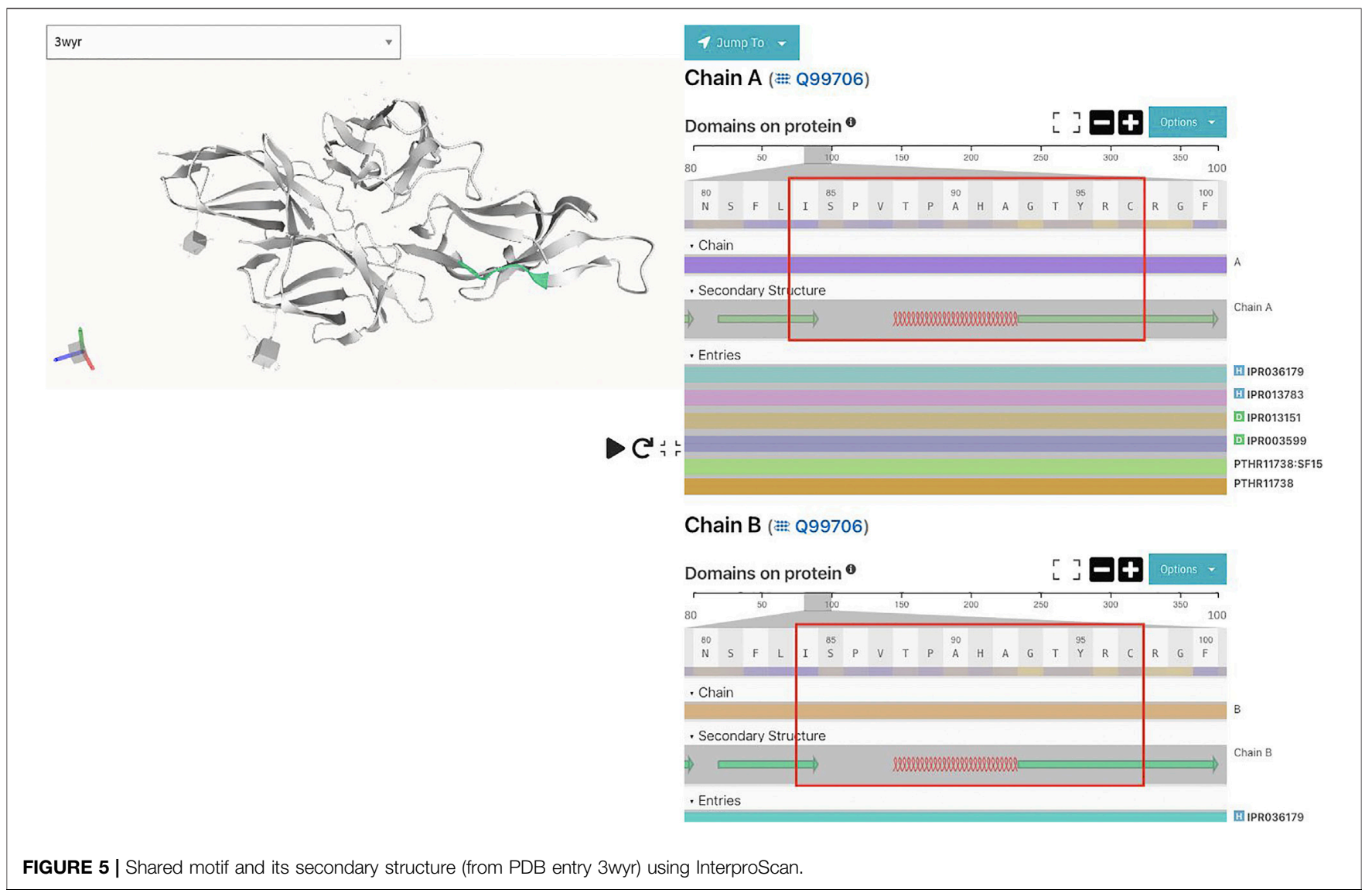

$$
\begin{gathered}
\mathrm{SP}=\frac{T N}{T N+F P}, \\
\text { Accuracy }=\frac{T P+T N}{T P+F N+T N+N P},
\end{gathered}
$$

where TN, TP, FN, and FP refer to the numbers of correctly predicted non-immunoglobulin proteins, correctly predicted immunoglobulin proteins, incorrectly predicted nonimmunoglobulin proteins, and incorrectly predicted immunoglobulin proteins, respectively. Sensitivity (SE) is also known as recall, and it measures the percentage that positive samples can be expected correctly over all the samples. SP indicators measure the probability of negative samples classified as non-immunoglobulins, and Accuracy is used to evaluate the overall performance of a prediction model.

\section{RESULTS AND DISCUSSION}

\subsection{Classification Results Under Different Features}

Props returned 93D best features, and the frequency of dipeptides $(\lambda$-gap $=0,1,2)$ is saved in features $1-75$, followed by 18 ACC features. The classification accuracy was $92.1 \%$ in the RF classifier and 10-fold cross-validation using Weka software. The MRMD method further reduced the dimension to 49D, and accuracy was 91.7\% using the same classifier. It can be seen that MRMD reduces nearly half of the feature dimension, but the accuracy is only dropped by $0.4 \%$ (Figure 2 ). After continuous attempts to reduce features, the optimal two features $\left(\mathrm{GC}^{\star}\right.$ and $\left.\mathrm{FC}^{*}\right)$ are finally obtained; the classification accuracy was $80.3 \%$ using the J48 classifier in Weka.

\subsection{D Features Scatter Distribution}

Figure 3 shows the scatter plot of $\mathrm{GC}^{\star}$ and $\mathrm{FC}^{\star}$ features. What stands out in Figure 3 is that immunoglobulin and nonimmunoglobulin samples can be distinguished. Immunoglobulins are scattered on the upper left with higher $\mathrm{FC}^{\star}$ values, and non-immunoglobulins are found in the lower right with higher $\mathrm{GC}^{\star}$ values. For 118 out of 119 nonimmunoglobulin samples, the $\mathrm{FC}^{\star}$ value is equal to or less than 5. Among these, the $\mathrm{FC}^{\star}$ value of 49 samples is zero. The $\mathrm{GC}^{\star}$ value for immunoglobulin samples is less than or equal to 12 .

\subsection{Interpretation of Feature FC*}

We noticed 49 out of 119 non-immunoglobulin samples had an $\mathrm{FC}^{\star}$ value of zero, whereas only four immunoglobulin samples had an $\mathrm{FC}^{\star}$ value of zero. Using motif search website MEME Suite 5.4.1 (Bailey and Elkan, 1994; Bailey et al., 2009) and running 109 immunoglobulin sequences, results showed that 107 out of 109 immunoglobulin samples had a motif, "ISNVTREDAGTYTC"

(Figure 4). Based on the reduced scheme, $\mathrm{Y}$ was treated as $\mathrm{F}$.

Immunoglobulin sequences were subjected to InterProScan (Zdobnov and Apweiler 2001) to understand the motif structure 
better to map protein domains. Results showed that the finding motif belonged to immunoglobulin subtype domain IPR003599.

Also, secondary structure predictions of the motif using JPred (Drozdetskiy et al., 2015) predict that the shared motif comprises alpha helices and beta sheets separated by disordered regions (Figure 5).

\section{CONCLUSION}

The present research aimed to examine a method to classify immunoglobulins and non-immunoglobulins using two features, $\mathrm{GC}^{\star}$ and $\mathrm{FC}^{\star}$. Classification of 228 samples (109 immunoglobulin samples and 119 non-immunoglobulin samples) revealed that the overall accuracy was $80.7 \%$ in the J48 classifier and 10 -fold crossvalidation using Weka software. The $\mathrm{FC}^{\star}$ feature identified in this study was found in immunoglobulin subtype domain IPR003599, which demonstrated that this extracted feature could represent functional and structural properties of immunoglobulins for forecasting.

\section{REFERENCES}

Anderson, M. J. (2001). A New Method for Non-parametric Multivariate Analysis of Variance. Austral Ecol. 26 (1), 32-46. doi:10.1111/j.14429993.2001.01070.pp.x

Ao, C., Zhou, W., Gao, L., Dong, B., and Yu, L. (2020). Prediction of Antioxidant Proteins Using Hybrid Feature Representation Method and Random forest. Genomics 112 (6), 4666-4674. doi:10.1016/j.ygeno.2020.08.016

Awais, M., Hussain, W., Rasool, N., and Khan, Y. D. (2021). iTSP-PseAAC: Identifying Tumor Suppressor Proteins by Using Fully Connected Neural Network and PseAAC. Cbio 16 (5), 700-709. doi:10.2174/ 1574893615666210108094431

Bailey, T. L., and Elkan, C. (1994). Fitting a Mixture Model by Expectation Maximization to Discover Motifs in Biopolymers. Proc. Int. Conf. Intell. Syst. Mol. Biol. 2, 28-36.

Bailey, T. L., Boden, M., Buske, F. A., Frith, M., Grant, C. E., Clementi, L., et al. (2009). MEME SUITE: Tools for Motif Discovery and Searching. Nucleic Acids Res. 37, W202-W208. doi:10.1093/nar/gkp335

Chen, X. X., Tang, H., Li, W. C., Wu, H., Chen, W., Ding, H., et al. (2016). Identification of Bacterial Cell Wall Lyases via Pseudo Amino Acid Composition. Biomed. Res. Int. 2016, 1654623. doi:10.1155/2016/1654623

Chen, Y., Ma, T., Yang, X., Wang, J., Song, B., and Zeng, X., (2021). MUFFIN: Multi-Scale Feature Fusion for Drug-Drug Interaction Prediction. Bioinformatics 37 (17), 2651-2658. doi:10.1093/bioinformatics/btab169

Cheng, Y., Gong, Y., Liu, Y., Song, B., and Zou, Q. (2021). Molecular Design in Drug Discovery: a Comprehensive Review of Deep Generative Models. Brief Bioinform 22 (6). doi:10.1093/bib/bbab344

Deng, L., Huang, Y., Liu, X., and Liu, H., (2021). Graph2MDA: a Multi-Modal Variational Graph Embedding Model for Predicting Microbe-Drug Associations. Bioinform. btab792. doi:10.1093/bioinformatics/btab792

Diener, C., Garza Ramos Martínez, G., Moreno Blas, D., Castillo González, D. A., Corzo, G., Castro-Obregon, S., et al. (2016). Effective Design of Multifunctional Peptides by Combining Compatible Functions. Plos Comput. Biol. 12 (4), e1004786. doi:10.1371/journal.pcbi.1004786

Ding, H., Liu, L., Guo, F.-B., Huang, J., and Lin, H. (2011). Identify Golgi Protein Types with Modified Mahalanobis Discriminant Algorithm and Pseudo Amino Acid Composition. Ppl 18 (1), 58-63. doi:10.2174/ 092986611794328708

Ding, Y., Yang, C., Tang, J., and Guo, F. (2021a). Identification of ProteinNucleotide Binding Residues via Graph Regularized K-Local Hyperplane Distance Nearest Neighbor Model. Berlin, Germany: Applied Intelligence.

\section{DATA AVAILABILITY STATEMENT}

Publicly available datasets were analyzed in this study. These data can be found here: DOI: $10.1039 / \mathrm{c} 5 \mathrm{mb} 00883 \mathrm{~b}$.

\section{AUTHOR CONTRIBUTIONS}

Conceptualization, HeW and GT; data collection or analysis, $\mathrm{HaW}, \mathrm{JZ}$ and YD; validation, HaW; writing-original draft preparation, HaW; writing - review and editing, HeW and GT. All authors have read and agreed to the published version of the manuscript.

\section{FUNDING}

The work was supported by the Special Science Foundation of Quzhou (2021D004).

Ding, Y., Tang, J., and Guo, F. (2021b). Protein Crystallization Identification via Fuzzy Model on Linear Neighborhood Representation. Ieee/acm Trans. Comput. Biol. Bioinf. 18 (5), 1986-1995. doi:10.1109/tcbb.2019.2954826

Dong, J., Zhao, M., Liu, Y., Su, Y., and Zeng, X., (2021). Deep Learning in Retrosynthesis Planning: Datasets, Models and Tools. Brief. Bioinform. bbab391. doi:10.1093/bib/bbab391

Dong, Q., Zhou, S., and Guan, J. (2009). A New Taxonomy-Based Protein Fold Recognition Approach Based on Autocross-Covariance Transformation. Bioinformatics 25 (20), 2655-2662. doi:10.1093/bioinformatics/btp500

Drozdetskiy, A., Cole, C., Procter, J., and Barton, G. J. (2015). JPred4: a Protein Secondary Structure Prediction Server. Nucleic Acids Res. 43 (W1), W389-W394. doi:10.1093/nar/gkv332

Feng, C., Ma, Z., Yang, D., Li, X., Zhang, J., and Li, Y. (2020). A Method for Prediction of Thermophilic Protein Based on Reduced Amino Acids and Mixed Features. Front. Bioeng. Biotechnol. 8, 285. doi:10.3389/fbioe.2020.00285

Feng, C., Zou, Q., and Wang, D. (2021). Using a Low Correlation High Orthogonality Feature Set and Machine Learning Methods to Identify Plant Pentatricopeptide Repeat Coding Gene/protein. Neurocomputing 424, 246-254. doi:10.1016/j.neucom.2020.02.079

Fu, X., Cai, L., Zeng, X., and Zou, Q. (2020). StackCPPred: a Stacking and Pairwise Energy Content-Based Prediction of Cell-Penetrating Peptides and Their Uptake Efficiency. Bioinformatics 36 (10), 3028-3034. doi:10.1093/ bioinformatics/btaa131

Gautam, A., Chaudhary, K., Kumar, R., Sharma, A., Kapoor, P., Tyagi, A., et al. (2013). In Silico approaches for Designing Highly Effective Cell Penetrating Peptides. J. Transl Med. 11, 74. doi:10.1186/1479-5876-11-74

Gong, Y., Peng, D., Liao, B., and Zou, Q., (2021). Accurate Prediction and Key Feature Recognition of Immunoglobulin. Appl. Sciences-Basel 11 (15), 6894. doi:10.3390/app11156894

Guo, Y., Yan, K., Lv, H., and Liu, B. (2021). PreTP-EL: Prediction of Therapeutic Peptides Based on Ensemble Learning. Brief. Bioinform. 22 (6), bbab358. doi:10.1093/bib/bbab358

Guo, Z., Wang, P., Liu, Z., and Zhao, Y. (2020). Discrimination of Thermophilic Proteins and Non-thermophilic Proteins Using Feature Dimension Reduction. Front. Bioeng. Biotechnol. 8, 584807. doi:10.3389/fbioe.2020.584807

Hansen, M., Kilk, K., and Langel, U. (2008). Predicting Cell-Penetrating Peptides. Adv. Drug Deliv. Rev. 60 (4-5), 572-579. doi:10.1016/j.addr.2007.09.003

He, S., Guo, F., Zou, Q., and Ding, H., (2020). MRMD2.0: A Python Tool for Machine Learning with Feature Ranking and Reduction. Curr. Bioinformatics 15 (10), 1213-1221. doi:10.2174/1574893615999200503030350

Hong, Z., Zeng, X., Wei, L., and Liu, X. (2020). Identifying Enhancer-Promoter Interactions with Neural Network Based on Pre-trained DNA Vectors and 
Attention Mechanism. Bioinformatics 36 (4), 1037-1043. doi:10.1093/ bioinformatics/btz694

Huang, S., He, X., Wang, G., and Bao, E., (2021). AlignGraph2: Similar GenomeAssisted Reassembly Pipeline for PacBio Long Reads. Brief Bioinform 22 (5), bbab022. doi:10.1093/bib/bbab022

Jiang, Q., Wang, G., Jin, S., Li, Y., and Wang, Y. (2013). Predicting Human microRNA-Disease Associations Based on Support Vector Machine. Ijdmb 8 (3), 282-293. doi:10.1504/ijdmb.2013.056078

Jin, Q., Meng, Z., Pham, T. D., Chen, Q., Wei, L., and Su, R. (2019). DUNet: A Deformable Network for Retinal Vessel Segmentation. Knowledge-Based Syst. 178, 149-162. doi:10.1016/j.knosys.2019.04.025

Khan, Y. D., Alzahrani, E., Alghamdi, W., and Ullah, M. Z., (2020). Sequence-based Identification of Allergen Proteins Developed by Integration of PseAAC and Statistical Moments via 5-Step Rule. Curr. Bioinformatics 15 (9), 1046-1055. doi:10.2174/1574893615999200424085947

Lepore, R., Olimpieri, P. P., Messih, M. A., and Tramontano, A. (2017). PIGSPro: Prediction of immunoGlobulin Structures V2. Nucleic Acids Res. 45 (W1), W17-W23. doi:10.1093/nar/gkx334

Li, H.-L., Pang, Y.-H., and Liu, B. (2021). BioSeq-BLM: a Platform for Analyzing DNA, RNA and Protein Sequences Based on Biological Language Models. Nucleic Acids Res. 49, e129. doi:10.1093/nar/gkab829

Liu, B., Gao, X., and Zhang, H. (2019). BioSeq-Analysis2.0: an Updated Platform for Analyzing DNA, RNA and Protein Sequences at Sequence Level and Residue Level Based on Machine Learning Approaches. Nucleic Acids Res. 47 (20), e127. doi:10.1093/nar/gkz740

Li, Q., Dong, B., Wang, D., and Wang, S. (2020a). Identification of Secreted Proteins from Malaria Protozoa with Few Features. Ieee Access 8, 89793-89801. doi:10.1109/access.2020.2994206

Li, Q., Zhou, W., Wang, D., Wang, S., and Li, Q. (2020b). Prediction of Anticancer Peptides Using a Low-Dimensional Feature Model. Front. Bioeng. Biotechnol. 8, 892. doi: $10.3389 /$ fbioe.2020.00892

Liu, Y., Wang, G., Wang, Y., and Huang, Y., (2020). A Deep Learning Approach for Filtering Structural Variants in Short Read Sequencing Data. Brief Bioinform 22 (4). doi:10.1093/bib/bbaa370

Lv, Z., Jin, S., Ding, H., and Zou, Q. (2019). A Random Forest Sub-golgi Protein Classifier Optimized via Dipeptide and Amino Acid Composition Features. Front. Bioeng. Biotechnol. 7, 215. doi:10.3389/fbioe.2019.00215

Manavalan, B., Basith, S., Shin, T. H., Wei, L., and Lee, G. (2019). Meta-4mCpred: A Sequence-Based Meta-Predictor for Accurate DNA 4mC Site Prediction Using Effective Feature Representation. Mol. Ther. - Nucleic Acids 16, 733-744. doi:10.1016/j.omtn.2019.04.019

Meng, C., Wu, J., Guo, F., Dong, B., and Xu, L. (2020). CWLy-Pred: A Novel Cell wall Lytic Enzyme Identifier Based on an Improved MRMD Feature Selection Method. Genomics 112 (6), 4715-4721. doi:10.1016/ j.ygeno.2020.08.015

Narciso, J. E. T., Uy, I. D. C., Cabang, A. B., Chavez, J. F. C., Pablo, J. L. B., PadillaConcepcion, G. P., et al. (2011). Analysis of the Antibody Structure Based on High-Resolution Crystallographic Studies. New Biotechnol. 28 (5), 435-447. doi:10.1016/j.nbt.2011.03.012

Naseer, S., Hussain, W., Khan, Y. D., and Rasool, N. (2021). NPalmitoylDeepPseaac: A Predictor of N-Palmitoylation Sites in Proteins Using Deep Representations of Proteins and PseAAC via Modified 5-Steps Rule. Cbio 16 (2), 294-305. doi:10.2174/1574893615999200605142828

Norman, R. A., Ambrosetti, F., Bonvin, A. M. J. J., Colwell, L. J., Kelm, S., Kumar, S., et al. (2020). Computational Approaches to Therapeutic Antibody Design: Established Methods and Emerging Trends. Brief. Bioinform. 21 (5), 1549-1567. doi:10.1093/bib/bbz095

Perez, E. E., Orange, J. S., Bonilla, F., Chinen, J., Chinn, I. K., Dorsey, M., et al. (2017). Update on the Use of Immunoglobulin in Human Disease: A Review of Evidence. J. Allergy Clin. Immunol. 139 (3), S1-S46. doi:10.1016/j.jaci.2016.09.023

Rahman, M. S., Rahman, M. K., Kaykobad, M., and Rahman, M. S. (2018). isGPT: An Optimized Model to Identify Sub-golgi Protein Types Using SVM and Random Forest Based Feature Selection. Artif. Intelligence Med. 84, 90-100. doi:10.1016/j.artmed.2017.11.003

Sanders, W. S., Johnston, C. I., Bridges, S. M., Burgess, S. C., and Willeford, K. O. (2011). Prediction of Cell Penetrating Peptides by Support Vector
Machines. Plos Comput. Biol. 7 (7), e1002101. doi:10.1371/ journal.pcbi.1002101

Schroeder, H. W., and Cavacini, L. (2010). Structure and Function of Immunoglobulins. J. Allergy Clin. Immunol. 125 (2), S41-S52. doi:10.1016/j.jaci.2009.09.046

Shao, J., and Liu, B. (2021). ProtFold-DFG: Protein Fold Recognition by Combining Directed Fusion Graph and PageRank Algorithm. Brief Bioinform 22 (3), bbaa192. doi:10.1093/bib/bbaa192

Shao, J., Yan, K., and Liu, B. (2021). FoldRec-C2C: Protein Fold Recognition by Combining Cluster-To-Cluster Model and Protein Similarity Network. Brief Bioinform 22 (3), bbaa144. doi:10.1093/bib/bbaa144

Solis, A. D. (2015). Amino Acid Alphabet Reduction Preserves Fold Information Contained in Contact Interactions in Proteins. Proteins 83 (12), 2198-2216. doi: $10.1002 /$ prot.24936

Song, G., Wang, G., Luo, X., Cheng, Y., Song, Q., Wan, J., et al. (2021). An All-ToAll Approach to the Identification of Sequence-specific Readers for Epigenetic DNA Modifications on Cytosine. Nat. Commun. 12 (1), 795. doi:10.1038/ s41467-021-20950-w

Song, B., Li, F., Liu, Y., and Zeng, X. (2021). Deep Learning Methods for Biomedical Named Entity Recognition: a Survey and Qualitative Comparison. Brief. Bioinform. 22 (6), bbab282. doi:10.1093/bib/bbab282

Susko, E., and Roger, A. J. (2007). On Reduced Amino Acid Alphabets for Phylogenetic Inference. Mol. Biol. Evol. 24 (9), 2139-2150. doi:10.1093/ $\mathrm{molbev} / \mathrm{msm} 144$

Tang, H., Chen, W., and Lin, H. (2016). Identification of Immunoglobulins Using Chou's Pseudo Amino Acid Composition with Feature Selection Technique. Mol. Biosyst. 12 (4), 1269-1275. doi:10.1039/c5mb00883b

Tang, Y.-J., Pang, Y.-H., and Liu, B. (2020). IDP-Seq2Seq: Identification of Intrinsically Disordered Regions Based on Sequence to Sequence Learning. Bioinformaitcs 36 (21), 5177-5186. doi:10.1093/bioinformatics/btaa667

Tang, Y.-J., Pang, Y.-H., and Liu, B., 2021, DeepIDP-2L: Protein Intrinsically Disordered Region Prediction by Combining Convolutional Attention Network and Hierarchical Attention Network. Bioinformatics 2021, btab810. doi:10.1093/bioinformatics/btab810

Tao, Z., Li, Y., Teng, Z., and Zhao, Y. (2020). A Method for Identifying Vesicle Transport Proteins Based on LibSVM and MRMD. Comput. Math. Methods Med. 2020, 8926750. doi:10.1155/2020/8926750

Wang, H., Ding, Y., Tang, J., and Guo, F. (2020). Identification of Membrane Protein Types via Multivariate Information Fusion with Hilbert-Schmidt Independence Criterion. Neurocomputing 383, 257-269. doi:10.1016/ j.neucom.2019.11.103

Wang, H., Tang, J., Ding, Y., and Guo, F. (2021). Exploring Associations of Noncoding RNAs in Human Diseases via Three-Matrix Factorization with Hypergraph-Regular Terms on center Kernel Alignment. Brief. Bioinformatics 22 (5), bbaa409. doi:10.1093/bib/bbaa409

Wang, J., Liu, X., Shen, S., and Deng, L. (2021). DeepDDS: Deep Graph Neural Network with Attention Mechanism to Predict Synergistic Drug Combinations. Brief. Bioinform. bbab390. doi:10.1093/bib/bbab390

Wang, X., Liu, J., Yang, Y., and Wang, G. (2021). The Stacking Strategy-Based Hybrid Framework for Identifying Non-coding RNAs. Brief Bioinform 22 (5). doi:10.1093/bib/bbab023

Wei, L., Liao, M., Gao, Y., Ji, R., He, Z., and Zou, Q. (2014). Improved and Promising Identification of Human MicroRNAs by Incorporating a HighQuality Negative Set. Ieee/acm Trans. Comput. Biol. Bioinf. 11 (1), 192-201. doi:10.1109/tcbb.2013.146

Yang, C., Ding, Y., Meng, Q., Tang, J., and Guo, F. (2021). Granular Multiple Kernel Learning for Identifying RNA-Binding Protein Residues via Integrating Sequence and Structure Information. Neural Comput. Appl. 33 (17), 11387-11399. doi:10.1007/s00521-020-05573-4

Yu, L., Su, Y., Liu, Y., and Zeng, X. (2021). Review of Unsupervised Pretraining Strategies for Molecules Representation. Brief. Funct. Genomics 20 (5), 323-332. doi:10.1093/bfgp/elab036

Zeng, X., Song, X., Ma, T., Pan, X., Zhou, Y., Hou, Y., et al. (2020). Repurpose Open Data to Discover Therapeutics for COVID-19 Using Deep Learning. J. Proteome Res. 19 (11), 4624-4636. doi:10.1021/acs.jproteome.0c00316

Zhai, Y., Chen, Y., Teng, Z., and Zhao, Y. (2020). Identifying Antioxidant Proteins by Using Amino Acid Composition and Protein-Protein Interactions. Front. Cel Dev. Biol. 8, 591487. doi:10.3389/fcell.2020.591487 
Zhang, J., Zhang, Z., Pu, L., Tang, J., and Guo, F. (2021). AIEpred: An Ensemble Predictive Model of Classifier Chain to Identify Anti-inflammatory Peptides. Ieee/acm Trans. Comput. Biol. Bioinf. 18 (5), 1831-1840. doi:10.1109/ tcbb.2020.2968419

Zhao, X., Wang, H., Li, H., Wu, Y., and Wang, G. (2021). Identifying Plant Pentatricopeptide Repeat Proteins Using a Variable Selection Method. Front. Plant Sci. 12, 506681. doi:10.3389/fpls.2021.506681

Zheng, L., Huang, S., Mu, N., Zhang, H., Zhang, J., Chang, Y., et al. (2019). RAACBook: a Web Server of Reduced Amino Acid Alphabet for Sequencedependent Inference by Using Chou's Five-step Rule. Database-the J. Biol. Databases Curation. baz131. doi:10.1093/database/baz131

Zou, Q., Zeng, J., Cao, L., and Ji, R. (2016). A Novel Features Ranking Metric with Application to Scalable Visual and Bioinformatics Data Classification. Neurocomputing 173, 346-354. doi:10.1016/j.neucom.2014.12.123

Conflict of Interest: Authors JZ and GT were employed by the company Geneis (Beijing) Co., Ltd.
The remaining authors declare that the research was conducted in the absence of any commercial or financial relationships that could be construed as a potential conflict of interest.

Publisher's Note: All claims expressed in this article are solely those of the authors and do not necessarily represent those of their affiliated organizations, or those of the publisher, the editors, and the reviewers. Any product that may be evaluated in this article, or claim that may be made by its manufacturer, is not guaranteed or endorsed by the publisher.

Copyright (C) 2022 Wan, Zhang, Ding, Wang and Tian. This is an open-access article distributed under the terms of the Creative Commons Attribution License (CC BY). The use, distribution or reproduction in other forums is permitted, provided the original author(s) and the copyright owner(s) are credited and that the original publication in this journal is cited, in accordance with accepted academic practice. No use, distribution or reproduction is permitted which does not comply with these terms. 